\title{
Comprehensive Medical System for Early Diagnosis of Rheumatoid Arthritis Based on Autoimmune Antibodies
}

\author{
Sha Liu (iD ${ }^{1,2}$ \\ ${ }^{1}$ Department of Rheumatology and Immunology, The First Hospital of Qiqihar, Qiqihar, 161000 Heilongjiang, China \\ ${ }^{2}$ Affiliated Qiqihar Hospital Southern Medical University, Qiqihar, 161000 Heilongjiang, China
}

Correspondence should be addressed to Sha Liu; 02238@qqhru.edu.cn

Received 21 January 2021; Accepted 24 April 2021; Published 17 May 2021

Academic Editor: Wenqing Wu

Copyright (C) 2021 Sha Liu. This is an open access article distributed under the Creative Commons Attribution License, which permits unrestricted use, distribution, and reproduction in any medium, provided the original work is properly cited.

\begin{abstract}
With the development of understanding of the pathogenesis of rheumatoid arthritis, it has led to the development of new treatment targets and new treatment guidelines. In the past 10 years, the treatment effect of RA patients has improved significantly. Due to the special clinical symptoms of RA, it is very important to have a clear assessment of the overall activity of the patient's disease and timely adjustment of treatment through joint monitoring of symptoms and laboratory indicators. Clinicians have always relied on clinical manifestations and imaging progress as the key basis for diagnosis. In addition, some markers that reflect disease activity are used to aid the diagnosis of RA, but in other autoimmune diseases, these markers are usually increased. The emergence of specific autoantibodies makes it easier for clinicians to distinguish RA from other autoimmune diseases and osteoarthritis. This study collected a large number of medical records through retrospective analysis, analyzed the epidemiological data, clinical characteristics, and laboratory indicators of RA patients, and analyzed the characteristics and laboratory indicators of patients in the stable disease group and active disease group. In order to further understand the clinical features, disease activity and related factors of RA in our country, and some new biomarkers for early rheumatoid arthritis, for example, the study found that the specificity of RF for the diagnosis of RA was $71.1 \%$, the sensitivity was $73.3 \%$, the combined detection of anti-CCP antibody + RF increased the specificity to $97.8 \%$, and anti-CCP the combined diagnosis of antibody+RF + GPI antigen increases the specificity of diagnosis to $100 \%$, which significantly reduces the sensitivity compared with all tests. These autoimmune antibodies can more effectively prevent the progression of the disease in the early diagnosis and treatment of rheumatoid arthritis, achieve long-term remission, and obtain very effective therapeutic effects.
\end{abstract}

\section{Introduction}

1.1. Background and Significance. In the early stage of rheumatoid arthritis, there is a "curing period" during which reasonable and effective treatment of the disease can prevent the progression of the disease, keep the patient in a state of asymptomatic for a long time, and effectively prevent joints. Therefore, early diagnosis of rheumatoid arthritis has become a prerequisite for early treatment and intervention to avoid poor prognosis. In recent years, many studies have been conducted on the early diagnosis of rheumatoid arthritis [1]. The application of magnetic resonance imaging is to see the pathological changes of early rheumatoid arthritis, and bone marrow edema is a special imaging of rheumatoid arthritis, which is very useful for the diagnosis of early rheu- matoid arthritis. At the same time, people have been trying to find specific markers of early rheumatoid arthritis, such as the widely used anticyclic citrullinated peptide antibody (CCP). Someone used protein chip technology to select early rheumatoid arthritis proteins for patients with early rheumatoid arthritis and control groups (normal healthy people and other patients who cause rheumatoid arthritis). As an objective and easy-to-use indicator, biological indicators have always attracted people's attention and are also a hot research topic [2]. In recent years, people have adopted a variety of methods to discover some new biomarkers in the early stages of rheumatoid arthritis, which provides a basis for the early diagnosis and treatment of RA, thereby improving joint function, improving work ability, and improving life. Quality provides a new method to diagnose early rheumatoid 
arthritis. Therefore, a comprehensive medical system designed to reduce the early diagnosis of autoimmune antibodies is very important for the treatment of rheumatoid arthritis by reducing doses, reducing toxic side effects, and improving curative effects [3].

1.2. Related Work Research. Vojdanian believes that although the current treatment prospects for rheumatoid arthritis have shown great improvement, many side effects have been reported. Within 12 weeks of M2000 treatment, RA index includes disease activity score (DAS28), simple disease activity index (SDAI) and C-reactive protein (CRP), erythrocyte sedimentation rate (ESR), rheumatoid factor (RF)), and anticirculation citrulline measured peptide antibodies against CCP and blood determinants. After 12 weeks of treatment with M2000, rheumatoid arthritis and migraine, which are considered inflammatory diseases, have been significantly improved. Therefore, it can be concluded that M2000, as a new type of nonsteroidal anti-inflammatory drug (NSAID) with immunosuppressive properties, can not only effectively treat rheumatoid arthritis but also treat migraine $[4,5]$.

Orsolini found that anticitrullinated protein antibody (ACPA) directly induces the differentiation and activation of osteoclasts, thus leading to bone loss around the joints. To analyze the effect of ACPA on the body bone mineral density (BMD) of established RA patients, 127 RA patients were recruited. In univariate analysis, ACPA-positive patients had lower BMD $Z$ scores at the femoral site (SD was lower than the average reference value for age and gender matching) $(p<0.01)$. A negative correlation between ACPA titer and BMD $Z$ score was observed at all sites $(p<0.01)$. Multivariate analysis adjusted for the main confounding variables confirmed the adverse effects of ACPA on the femoral site $(p<0.05)$, but no effect on lumbar spine BMD. ACPA has a negative potency dependence on the BMD of the femur, which is mainly composed of the cortical bone. ACPApositive patients, especially those with high titers, should undergo bone examination and receive treatment with bone protectants. Antirheumatic drugs that reduce the ACPA titers that can change the disease may have a positive impact on systemic bone mass $[6,7]$.

\subsection{Innovation}

(1) This paper studies the detection of serum anti-PDI4 antibody levels and found that the level and positive rate of anti-PLD4 antibodies in the PA group are higher than those in the non-RA group and healthy controls, indicating that the antibody RAD4 is an autoantibody for rheumatoid arthritis and can be used for serology The new effective markers detected are used for the diagnosis and differential diagnosis of RA

(2) This paper found that in the detection of RA autoantibodies, RF is a diagnostic marker with lower specificity and sensitivity than anti-CCP antibodies. The combination of RF, anti-CCP antibody, AKA, and anti-PADI4 antibody can provide a lot of clinical diagnostic information
(3) In this article, by detecting the level of DKK-1 in the serum of patients with rheumatoid arthritis and healthy individuals, and analyzing its correlation with the results of clinical trials, bone density determination, and other related tests, the expression level of DKK-1 in the serum of RA patient preliminary exploration and related clinical significance provides clinical evidence for the clinical application of DKK-1

\section{Early Diagnosis of Autoimmune Antibodies}

2.1. Autoimmune Antibody. The autoantibodies produced by humoral immunity are not only related to the onset and severity of the disease. Compared with the diagnosis of RA, the importance of its research is irreplaceable. The new guidelines for the diagnosis and treatment of rheumatoid arthritis indicate that the goal of RA treatment is to achieve clinical relief and control the destruction of cartilage and subchondral bone after early joint hyperplasia. Therefore, rheumatoid arthritis is mainly based on timely and accurate diagnosis and treatment to effectively prevent irreversible joint damage [7]. In 1987, the American College of Rheumatology (ACR) diagnostic RA classification criteria were widely used in the diagnosis of RA, mainly focusing on clinical manifestations. However, early patients with RA do not have specific symptoms; so, its criteria are not suitable for early diagnosis of RA [8].

Searching for sensitive and specific indicators in the serum of patients with early rheumatoid arthritis has always been a hot spot for researchers at home and abroad. Previous indicators include rheumatoid factor, C-reactive protein (CRP), and red blood cell sedimentation rate. In recent years, anticyclic yellow peptide (CCP) antibodies have become the subject of extensive research. In 2009, the new diagnostic criteria of rheumatology in the United States for RA included the above four serological indicators into the diagnostic criteria [9]. The detection of autoantibodies in rheumatoid arthritis has always been a hot spot for researchers, and its research progress mainly includes the following aspects.

(1) Rheumatoid factor (RF). RF was first discovered in 1927 and was one of the first important serological markers for the diagnosis of RA. RF is synthesized in the plasma cells of the synovium and is mainly an autoantibody against epitopes in the Fc fragment of Ig molecules in serum

(2) Antiperinuclear factor (APF). This substance is a protein in the form of IgG in human serum. APF is related to the condition of RA patients. Its appearance suggests a poor prognosis, but many reasons limit its clinical application

(3) Antikeratin antibody (AKA). The study found that there is no difference between AKA and RF and can be detected in the early serum of patients. Therefore, AKA provides a powerful indication for the diagnosis of early RA patients who are negative for RF and has been widely used clinically 
TABle 1: Calculation formulas for sensitivity, specificity, positive predictive value, and negative predictive value.

\begin{tabular}{lccc}
\hline Test & Case & Noncase & Total \\
\hline Positive & $w$ (true positive) & $x$ (false positive) & $w+x$ \\
Negative & $y$ (false negative) & $z$ (true negative) & $y+z$ \\
Total & $w+y$ & $x+z$ & $w+x+y+z$ \\
\hline
\end{tabular}

(4) Antifilaggrin antibody (AFA). Experiments have shown that AFA appears in the serum of patients with early RA, even earlier than anti-CCP antibodies, and is related to disease activity

(5) Anti-RA33 antibody. It can appear in the serum of early RA patients and is a good indicator of serological diagnosis. It has nothing to do with rheumatoid factor. Detection of anti-PA33 in early RA patients with negative atypical rheumatoid factor can help improve diagnosis and achieve early diagnosis and treatment

(6) Anticyclic citrullinated peptide (CCP) antibody. Internal and external comparisons show that the diagnostic specificity of anti-CCP antibodies is relatively certain, but there are some differences in sensitivity. Due to its high diagnostic value, anti-CCP antibodies have been a hot spot for scholars in recent years

(7) Peptidyl arginine deiminase 4 (PADI4). The specificity is $99 \%$, which is the most specific serological diagnostic index of RA, which can occur before the onset of RA; so, it can be used for the early diagnosis of RA

(8) Comprehensive detection of rheumatoid arthritis. Many researchers have conducted a lot of research on the combined detection of RF and anti-CCP antibodies, seeking a logical combination of diagnostic experiments to provide a rich and accurate scientific experimental basis for the diagnosis of clinical diseases [10], so as to achieve accurate diagnosis and reasonable treatment

2.2. Rheumatoid Arthritis. Rheumatoid arthritis (RA) is a common clinical autoimmune disease that occurs in the joints of the limbs. It mainly manifests as repeated chronic inflammation of the joints. In advanced patients, joint deformities and disabilities can even lead to serious complications of the heart, skin, and other tissues and organs. In my country, the incidence of RA is about $0.35 \%$, and the incidence of women is still very high, with about 4 million patients. The etiology of RA is complex, and the high incidence may be related to many factors, such as infection stimulation, genetic factors, internal and external environmental changes, immune diseases, and smoking $[11,12]$.

Rheumatoid arthritis is a systemic disease of chronic immune system disease. Worldwide, the incidence of rheumatoid arthritis is about $1 \%$, and the ratio of men to women is $2.5 / 1$. The high incidence of this disease is between 40 and 70 years old. With age, there is an upward trend. The disease is characterized by joint pain, swelling, stiffness and destruction of bone and cartilage tissue, and in severe cases even loss of mobility, resulting in a severe reduction in the quality of life of the patient and a reduction in the life expectancy of the patient. The pathological changes of rheumatoid arthritis are mainly the proliferation of joint cells in the joint cavity, inflammatory cells that erode inflammatory joints, and the formation of new blood vessels in the joint joint cavity, leading to joint damage and deformity $[13,14]$.

Most patients with rheumatoid arthritis have chronic relapsing episodes, and some patients develop rapidly within 1-2 years. The etiology of rheumatoid arthritis is not fully understood. Many studies have been conducted on the pathogenesis of rheumatoid arthritis, but it is still unclear. Current research has found that rheumatoid arthritis is related to genetic factors, infectious factors, sex hormones, and the environment. The pathogenesis of rheumatoid arthritis is based on susceptibility genes and immune disorders caused by pathogens such as the environment and infection. These diseases can cause autoimmune reactions and cause inflammatory damage to joint tissues [15].

Early diagnosis and treatment of rheumatoid arthritis are very important, because the damage to the joint structure of patients with rheumatoid arthritis is irreversible [16]. It is important to be able to detect the disease as soon as possible and treat it with standard therapies so that the disease can be well controlled. Delay or even prevent the progression of the disease, prevent joint damage, avoid the poor prognosis of patients with disability or death, and strive to enable patients to enjoy a higher quality of life [17].

2.3. Diagnostic Criteria for RA. The diagnosis of RA refers to the classification standard and scoring system proposed by ACR (American Association of Rheumatology) and EULAR (European Alliance against Rheumatism). That is to say, there is at least 1 joint swelling and pain, there is evidence of arthritis (clinical or ultrasound or magnetic resonance imaging), arthritis caused by other diseases is excluded, and there is a typical routine RA radiological change or meet the RA classification criteria. According to the scoring system, a total score of 6 is diagnosed as RA $[18,19]$, as shown in Table 1.

Sensitivity refers to the percentage of confirmed cases that are also judged positive through evaluation [20]. The ideal value is $100 \%$. The calculation formula is

$$
w /(w+y) \times 100 \%
$$

Specificity refers to the percentage of confirmed noncases that are also judged negative by the evaluation test, and the ideal value is $100 \%$. The calculation formula is

$$
z /(x+z) \times 100 \%
$$

The positive prognostic value refers to the ratio of true positive individuals to the number of positive test results. The calculation formula of the measured value is

$$
w /(w+x) \times 100 \%
$$


TABLE 2: Comparison of antibody, antigen, and concentration of each group.

\begin{tabular}{lccc}
\hline Detection Indicator & RA group & Non-RA group & Control group \\
\hline Anti-MCV antibody $(\mathrm{U} / \mathrm{mL})$ & $268.9 \pm 125.36$ & $12.44 \pm 3.89$ & $10.01 \pm 3.08$ \\
GPI antigen $(\mathrm{mg} / \mathrm{L})$ & $1.211 \pm 0.528$ & $0.13 \pm 0.06$ & $0.087 \pm 0.033$ \\
Anti-CCP antibody $(\mathrm{RU} / \mathrm{mL})$ & $719.43 \pm 477.15$ & $13.66 \pm 4.41$ & $11.2 \pm 3.55$ \\
RF $(\mathrm{IU} / \mathrm{mL})$ & $144.20 \pm 81.68$ & $13.72 \pm 5.23$ & $8.16 \pm 2.96$ \\
\hline
\end{tabular}

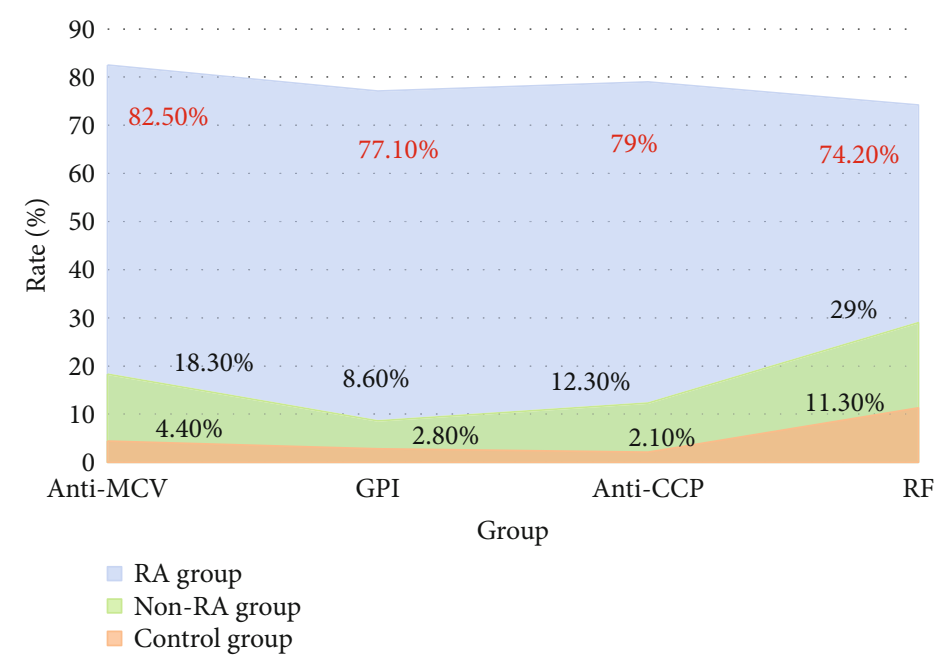

FIGURE 1: Comparison of antibody, antigen, and positive rate of each group.

TABLE 3: Comparison of antibody, antigen and positive rate of each group.

\begin{tabular}{lccc}
\hline Detection indicator & $\begin{array}{c}\text { RA } \\
\text { group }\end{array}$ & $\begin{array}{c}\text { Non-RA } \\
\text { group }\end{array}$ & $\begin{array}{c}\text { Control } \\
\text { group }\end{array}$ \\
\hline $\begin{array}{l}\text { Anti-MCV antibody } \\
\text { (U/mL) }\end{array}$ & $82.5 \%$ & $18.3 \%$ & $4.4 \%$ \\
$\begin{array}{l}\text { GPI antigen (mg/L) } \\
\begin{array}{l}\text { Anti-CCP antibody } \\
\text { (RU/mL) }\end{array}\end{array}$ & $77.1 \%$ & $8.6 \%$ & $2.8 \%$ \\
RF $(\mathrm{IU} / \mathrm{mL})$ & $79 \%$ & $12.3 \%$ & $2.1 \%$ \\
\hline
\end{tabular}

Negative predictive value refers to the percentage of true negative individuals whose test results are negative. The formula for calculating the predicted value is

$$
z /(y+z) \times 100 \%
$$

The DAS28 scoring standard widely used in clinical practice is used to determine the degree of RA activity [21]. DAS28 rating refers to the PREVOO calculation method, which is based on the DAS28 rating calculation formula for 28 joints:

DAS28 $=[0.56 \times \operatorname{sqrt}(t 28)+0.28 \times \operatorname{sqrt}(s w 28)+0.70 \times \operatorname{Ln}($ SER $)] \times 1.08+0.16$
2.4. Peak Admission for Rheumatoid Arthritis. Collect the clinical data of 2500 RA patients admitted to the rheumatology department in the last 3 years, carry out periodic statistical analysis, calculate the peak period of admission for rheumatoid arthritis, and summarize the seasonality of admission for rheumatoid arthritis. It not only provides an epidemiological basis for the clinical prevention and treatment of RA, but also improves the ability of traditional Chinese medicine to cure the disease. At the same time, it also provides scientific statistical methods for time medicine and provides a scientific basis for our country to understand the relationship between time and disease [22, 23]. According to the season, patients with rheumatoid arthritis were divided into four groups, namely, the spring group (631 people), the summer group (572 people), the autumn group (628 people), and the winter group (669 people). The statistical method uses the periodic distribution method to first determine the average cumulative number of days in each season, convert it to an angle, take the sine and cosine values, and then multiply by the group frequency (number of patients). Then, use the formula

$$
\begin{gathered}
x=\sum f \cos \alpha / n, \\
y=y \sum f \sin \alpha / n, \\
r=\sqrt{x^{2}+y^{2}}, \\
\alpha=\operatorname{arctgy} / x .
\end{gathered}
$$




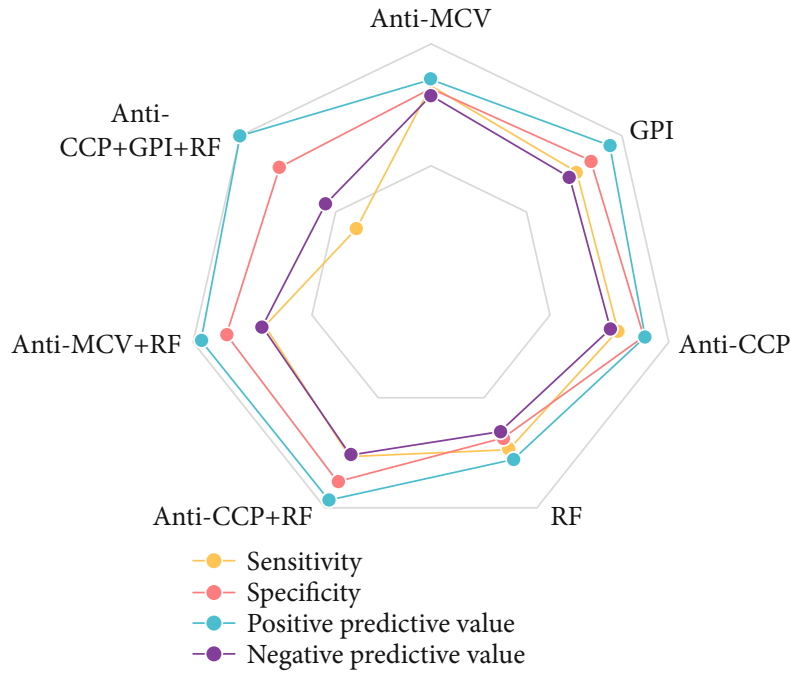

FIgURE 2: Sensitivity and specificity of serum markers in RA.

Simplified conversion is available:

$$
\begin{aligned}
& \alpha=\operatorname{arctg} \sum \sin \alpha / \sum \cos \alpha, \\
& s=(180 / \pi) \sqrt{-2 \ln r} .
\end{aligned}
$$

Look up the table, calculate the angle mean $\alpha$ and standard deviation $s$, convert them to the corresponding time, perform a significant test on the concentration vector $r$, and get the peak of the introduction of rheumatoid arthritis [24, 25].

2.5. Slice Image Feature Extraction. First, we observed a large number of neural slice images. The shapes of different types of neural function packages in the nerve slice images do not show specific feature rules. However, in actual situations, according to the doctor's professional introduction, it shows different types of nerve function keys. Shape cannot be used as a feature evaluation parameter, and nerve bundles of the same type can also have different shapes. Therefore, this article does not take the form of graph as the evaluation parameter, but chooses internal features to represent it [26]. Since the color feature difference between different types of nerve function bundles is very small, it cannot be used as an effective evaluation parameter. In this way, we choose local texture as the internal feature of the neural slice image, and the choice of texture feature will directly affect the result of subsequent recognition. Based on the following evaluation parameters, the texture features of the image stained by neurofetus are calculated $[27,28]$.

The average gray value reflects the average gray value of the image

$$
m=\sum_{i=0}^{L-1} i p(i)
$$

Variance reflects the discrete distribution of image gray levels.

$$
\beta=\sqrt{\sum_{i=0}^{L-1}(i-m)^{2} p(i) .}
$$

Kurtosis reflects the approximate state of the gray image distribution when it is close to the mean. It is used to judge whether the grayscale distribution of the image is too concentrated near the middle grayscale. The smaller the kurtosis, the more concentrated it is.

$$
\omega=\frac{1}{\beta^{4}} \sum_{i=0}^{L-1}(i-m)^{2} p(i)-3 .
$$

Smoothness is a measure of the relative smoothness of brightness in an area. For areas with constant brightness, the change is small, and so $T$ is equal to zero. For areas with large deviations in grayscale values, if the change is large, $T$ is close to 1 .

$$
T=1-\frac{1}{1+\beta^{2}}
$$

The principle of average filtering is to select a template composed of several adjacent pixels, find the average value of all pixels in the template, and then match the average value of the current pixel with the gray level of the processed image, which is

$$
g(x, y)=\frac{1}{m} \sum f(x, y)
$$

The basic design idea of the median filter is to replace the value of a point in the digital image or sequence with the average value of each point near the point and then change the pixels with a large difference in the gray value of the surrounding pixels to be close to the surrounding pixels. Therefore, a single noise point can be eliminated.

$$
Y(n)=\operatorname{med}[X(n-N) \cdots X(n) \cdots X(n+N)] .
$$

\section{Comprehensive Medical System for Early Diagnosis of Autoimmune Antibodies}

3.1. Test Subject. From March 2019 to September 2019, select RA patients, non-RA patients, and health examiners in orthopedic hospitals and record the clinical and laboratory data of the patients, such as gender, age, course of disease, joint performance, erythrocyte sedimentation rate, and Creactive protein etc. The fasting venous blood of the patient was collected. The above data and samples were collected under the informed consent of the patient and the approval of the competent committee.

\subsection{Test Design}


TABLE 4: Sensitivity and specificity of serum markers in RA.

\begin{tabular}{lcccc}
\hline Index & Sensitivity & Specificity & Positive predictive value & Negative predictive value \\
\hline Anti-MCV antibody & $82.9 \%$ & $81.8 \%$ & $85.5 \%$ & $78.8 \%$ \\
GPI antigen & $76.3 \%$ & $95.7 \%$ & $93.7 \%$ & $72.7 \%$ \\
Anti-CCP antibody & $78.6 \%$ & $89.5 \%$ & $90.1 \%$ & $75.5 \%$ \\
RF & $73.5 \%$ & $68.2 \%$ & $78 \%$ & $65.3 \%$ \\
Anti-CCP antibody+RF & $76.7 \%$ & $88.1 \%$ & $96.5 \%$ & $76 \%$ \\
Anti-MCV antibody+RF & $69.8 \%$ & $86 \%$ & $96.6 \%$ & $70.9 \%$ \\
Anti-CCP antibody+GPI antigen+RF & $39 \%$ & $79.3 \%$ & $100 \%$ & $55.2 \%$ \\
\hline
\end{tabular}

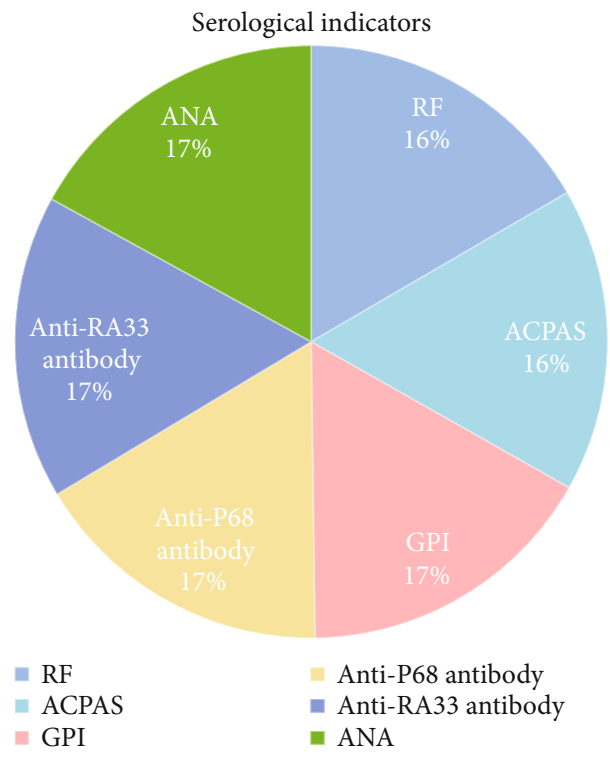

Figure 3: Related serological indicators.

(1) Sample collection. All subjects in the study collect 3$5 \mathrm{~mL}$ of venous blood on an empty stomach, centrifuge at $3000 \mathrm{r} / \mathrm{min}$ for $5-10$ minutes, and collect the supernatant. Store in a low-temperature refrigerator at $-70^{\circ} \mathrm{C}$ and determine the batch after the freezethaw cycle

(2) ELISA test. Anti-CCP antibody, anti-MCV antibody, and serum GPI antigen concentration were all measured by the double antibody ELISA sandwich method

(3) Take out the 96-well plate coated with different antibodies purchased from the reagent company from the refrigerator and place it at room temperature. Add $100 \mu \mathrm{l}$ of diluted test serum, negative control, positive control, and reference standard to 96-well wells

(4) Incubate for 30 or 60 minutes in a water bath at room temperature or $37^{\circ} \mathrm{C}$. Empty the 96 -well plate, wash it 5 times with the predetermined washing solution, and then dry it with clean absorbent paper

(5) Add $100 \mu \mathrm{l}$ of enzyme-labeled antibody solution to each well. Incubate for 15 or 60 minutes in a water bath at room temperature or $37^{\circ} \mathrm{C}$. The 96 -well plate is washed, washed again , and dried

(6) Add $100 \mu \mathrm{l}$ of substrate solution to each well, incubate at room temperature for 15 minutes, and add $100 \mu \mathrm{l}$ of reaction stop solution to each well

(7) Rate nephelometry detection. The turbidimetric method is used for automatic RF detection in serum

Use SPSS13.0 statistical software for statistical analysis. Use a four grid table to calculate the sensitivity, specificity, positive predictive value, and negative predictive value of each test index. For comparison between groups or between groups, the enumeration data uses the second test, the normal distribution measurement data uses the $t$-test to compare the two groups, and the distortion distribution uses the nonparametric test. Spearman correlation analysis was used to compare the correlation between serum markers and the DAS28 score of RA disease activity. $p<0.05$ was regarded as a statistically significant standard.

3.3. Experimental Results. The anti-MCV antibody, GPI antigen, and anti-CCP serum levels of the three groups of subjects were calculated by ELISA technology. Rate nephelometry was used to determine the serum RF concentration of three groups of individuals. The results showed that compared with the other two groups, the concentrations of anti-MCV antibody, GPI antigen, anti-CCP antibody, and $\mathrm{RF}$ in the RA group were significantly higher $(p<0.05)$. Comparing the non-RA group and the normal group, there was no significant difference in the concentration of the four serum markers $(p>0.05)$, as shown in Table 2 .

\section{Effect of the Early Diagnosis System of Autoimmune Antibodies}

4.1. Comparison of Positive Rates of Serum Markers. There are relatively few reports on the combination of anticitrulline antibody and GPI antigen for the diagnosis of RA. This article analyzes and compares the four sera by measuring the concentration of anti-MCV antibody, anti-CCP antibody, GPI antigen, and RF in the serum of patients diagnosed with RA. The sensitivity, specificity, and relevance of markers are in the clinical diagnosis of RA. The positive crisis standard of each serum marker is the positive standard of anti-MCV antibody which is $>20 \mathrm{U} / \mathrm{mL}$. The positive standard for CPI 
TABLE 5: Related serological indicators.



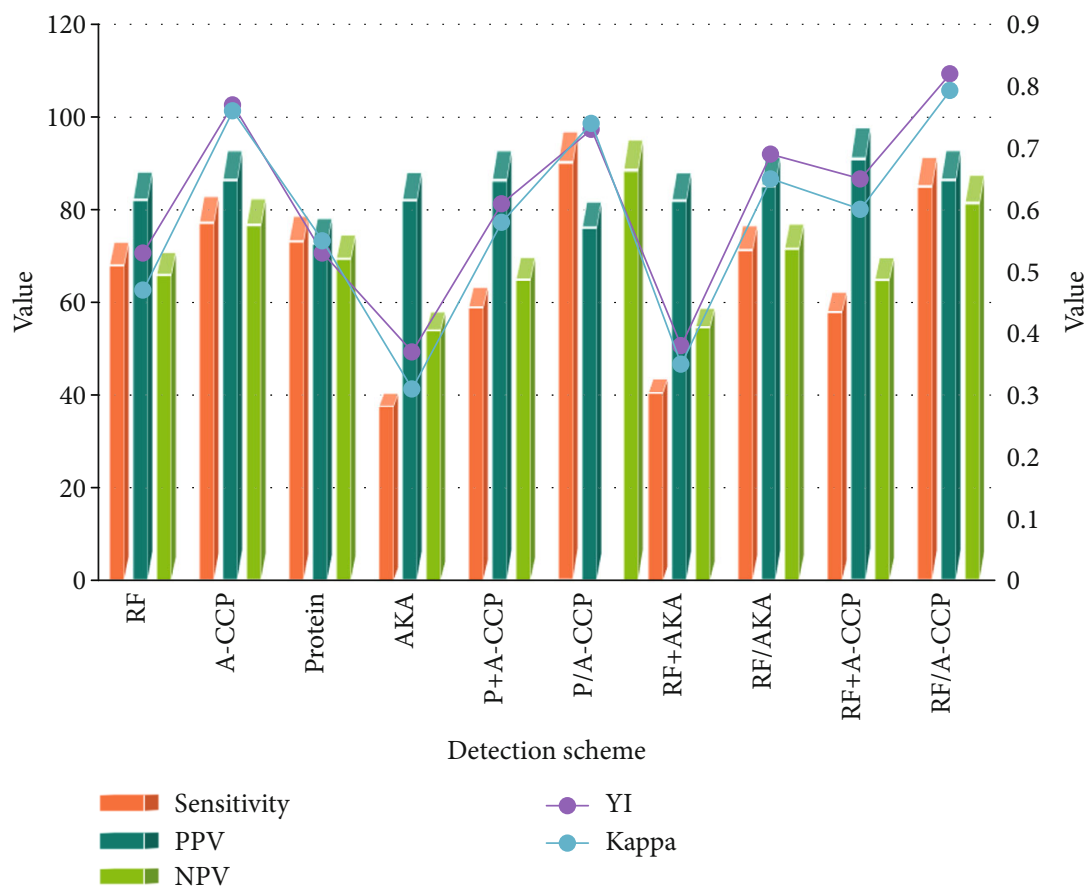

FIGURE 4: Comparison of single and combined testing schemes for 4 indicators. 
TABLE 6: Comparison of single and combined testing schemes for 4 indicators.

\begin{tabular}{|c|c|c|c|c|c|c|c|}
\hline Test items & Sensitivity & Specificity & PPV & NPV & Accuracy & YI & Kappa \\
\hline $\mathrm{RF}$ & 73.4 & 89.8 & 88.6 & 71.2 & 75.03 & 0.53 & 0.47 \\
\hline Anti-CCP antibody & 83.3 & 92.5 & 93.2 & 82.8 & 88 & 0.77 & 0.76 \\
\hline $14-3-3 \eta$ & 79 & 74.1 & 78.5 & 75 & 76.9 & 0.53 & 0.55 \\
\hline AKA & 40.7 & 94.2 & 88.5 & 58.3 & 65 & 0.37 & 0.31 \\
\hline $14-3-3 \eta+$ anti-CCP antibody & 63.65 & 95.64 & 93.18 & 70.14 & 78.56 & 0.61 & 0.58 \\
\hline 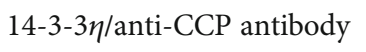 & 97.3 & 72.61 & 82.16 & 95.77 & 86 & 0.73 & 0.74 \\
\hline $\mathrm{RF}+\mathrm{AKA}$ & 43.85 & 94.35 & 88.42 & 59.07 & 66.22 & 0.38 & 0.35 \\
\hline $\mathrm{RF} / \mathrm{AKA}$ & 76.91 & 93 & 91.86 & 77.32 & 86.12 & 0.69 & 0.65 \\
\hline $\mathrm{RF}+$ anti-CCP antibody & 62.59 & 99 & 98.16 & 70.07 & 79.36 & 0.65 & 0.601 \\
\hline RF/anti-CCP antibody & 91.78 & 90.39 & 93.23 & 87.92 & 90.08 & 0.82 & 0.793 \\
\hline
\end{tabular}

TABLE 7: Serum concentration of each group.

\begin{tabular}{lccc}
\hline Group & ZNF706 & WIBG & GABAPAPL2 \\
\hline ERA & $86.35+2.96$ & $1539.12 \pm 116.42$ & $39.47 \pm 2.86$ \\
NERA & $60.37+2.19$ & $1233.67 \pm 99.34$ & $28.63 \pm 1.76$ \\
CTD & $52.64 \pm 3.91$ & $993.14 \pm 85.91$ & $20.68 \pm 0.94$ \\
Healthy & $47.35 \pm 2.64$ & $424.52 \pm 30.96$ & $15.03 \pm 0.55$ \\
\hline
\end{tabular}

antigen is $>0.20 \mathrm{mg} / \mathrm{L}$. The positive standard for CCP antibody is $>25 \mathrm{RU} / \mathrm{mL}$. The positive RF standard is $>15 \mathrm{IU} / \mathrm{mL}$. The positive rate of the four serum markers in the RA group was significantly higher than that of the non-RA group and the control group, and the difference was statistically significant $(p<0.05)$, as shown in Figure 1, Table 3.

4.2. Comparison of Sensitivity and Specificity of Serum Markers in RA. The sensitivity of the four detection markers from high to low is anti - MCV antibody $(82.9 \%)>$ anti CCP antibody $(78.6 \%)>$ GPI antigen $(76.3 \%)>\mathrm{RF}(73.5 \%)$, of which anti-MCV antibody is (82.9\%). The sensitivity is the highest, significantly higher than RF $(p<0.05)$, and there is no significant difference between other indicators $(p>0.05$ ). The specificity of the four detection markers from high to low is GPI antigen $(95.7 \%)>$ anti - CCP antibody $(89.5 \%)$ $>$ anti - MCV antibody (81.8\%) > RF (68.2\%), among which is GPI antigen and anti-CCP antibody. The specificity of RF was significantly higher than that of RF $(p<0.01$ and $<$ $0.05)$, and the specificity among other markers was not statistically significant $(p>0.05)$. The positive predictive value of the four test markers was GPI antigen $(93.7 \%)>$ anti - CCP antibody $(90.1 \%)>$ anti - MCV antibody $(85.5 \%)>\mathrm{RF}(78$ $\%)$. The negative predictive value from high to low is antiMCV antibody $(78.8 \%)>$ anti - CCP antibody $(75.5 \%)>$ GPI antigen $(72.7 \%)>\mathrm{RF}(65.3 \%)$. In the comparison of the positive predictive value, the value of GPI antigen was significantly higher than that of RF $(p<0.05)$, and there was no statistical significance among other indicators $(\mathrm{P}>0.05)$. There was no statistical difference in the negative prognostic value of each index $(p>0.05)$, as shown in Figure 2 and Table 4.
4.3. Serological Indicators Related to Rheumatoid Arthritis. RA cannot be cured, but early diagnosis can control RA patients as soon as possible and use drugs in a timely and reasonable manner, thereby significantly improving the quality of life of patients. ACR and the European Union Against Rheumatism (EULAR) jointly proposed a new RA diagnostic model in 2009, including a new laboratory-labeled anticyclic citrullinated peptide (CCP) antibody. The combined detection of the two can improve the efficiency of early diagnosis of rheumatoid arthritis. With the deepening of research, domestic and foreign researchers gradually discovered some new indicators and detection methods. Some of them have higher sensitivity, some have stronger specificity, some are closely related to the development and prognosis of the disease, and some can be found in the early stages of the disease or even long before clinical diagnosis. With the continuous emergence of new detection markers, understanding the pros and cons of different markers in diagnosis and complementarity is what clinicians need in clinical practice. ACPAS is a large class of autoantibodies important for the diagnosis of RA, including anti-MCV antibodies, anti-cyclic citrullinated peptide antibodies (CCP) antibodies, anti-perinuclear factor antibodies (APF), and anti-keratin antibodies (AKA), anti-keratin-filaggrin antibody (AFA), and anti-citrullinated fibrinogen antibody (ACF), as shown in Figure 3 and Table 5.

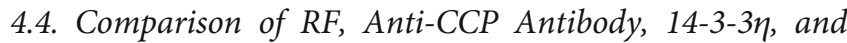
AKA Detection Protocols. Finding high-sensitivity and highspecificity laboratory diagnostic methods [29], especially early diagnostic indicators, has always been the focus of laboratory diagnosis. In the research of RA autoantibodies, we have experienced RF, AKA, anti-CCP antibodies, and so on. The accuracy and value of YI and Kappa are comprehensive statistical parameters of sensitivity and specificity. Generally, the higher the YI, the better the authenticity of the measurement. The higher the accuracy, the better the consistency between the test result and the actual value. Kappa analysis examined the influence of random factors on cohesion. The higher the Kappa value, the better the reliability of the diagnostic test [30]. The four indicators were combined in two projects in parallel, and the parameters of a total of twelve combinations were analyzed. When the dual target detection is parallel, the highest sensitivity of the combined 


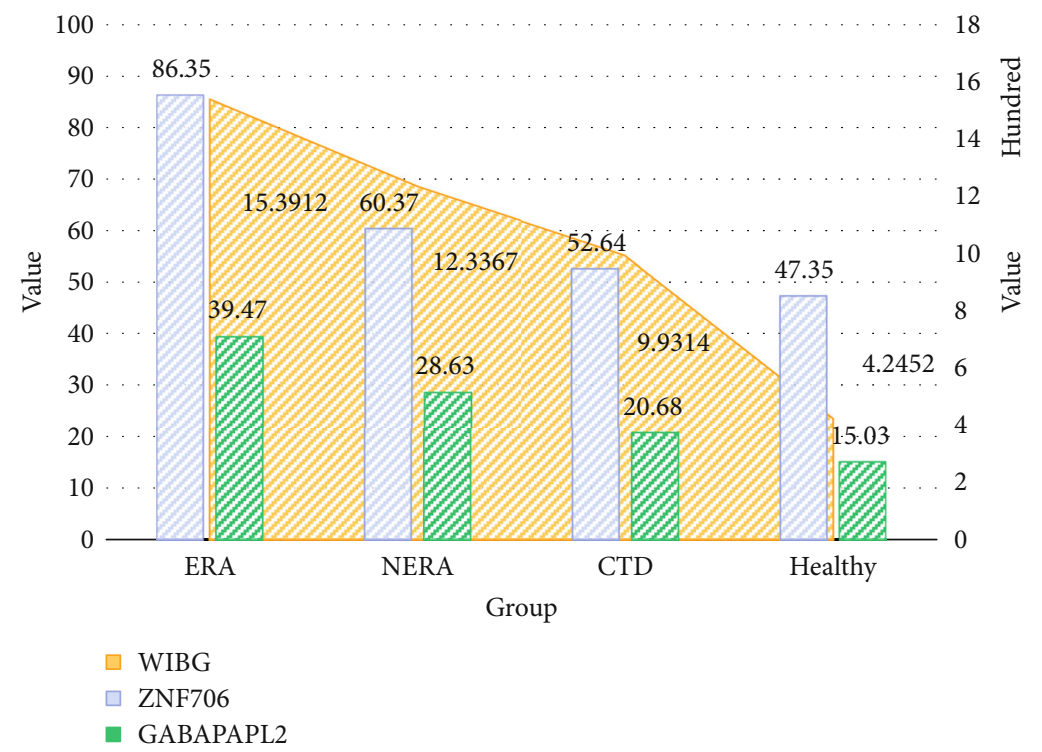

FIGURE 5: Serum concentration of each group.

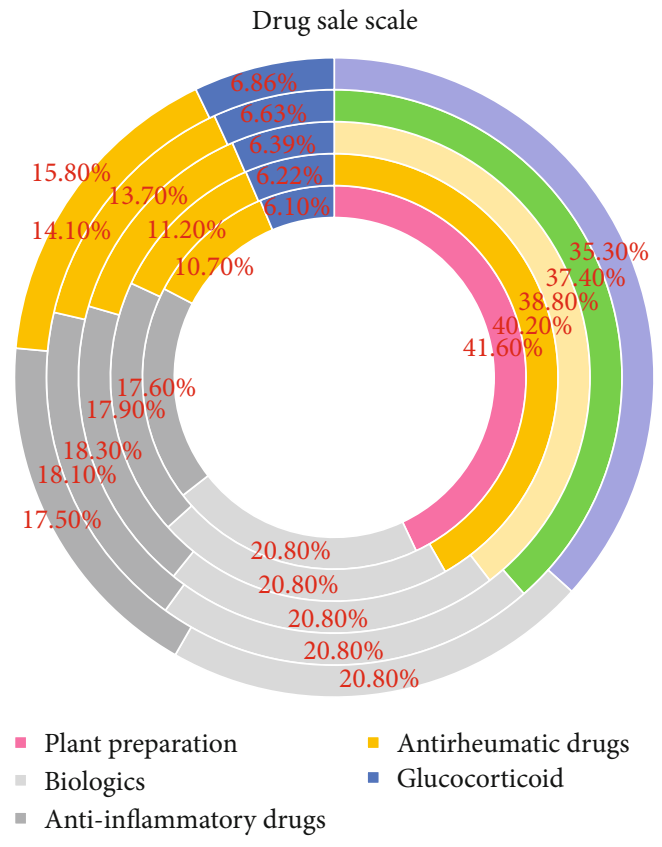

FIgURE 6: Proportion of drugs in recent years.

14-3-3 protein detection (shown as protein in the figure) and anti-CCP antibody is $96.81 \%$, but the specificity of this combination is only $73.75 \%$, positive predictive value. The negative predictive value is $81.25 \%$, the negative predictive value is $95.16 \%$, the accuracy is $86.21 \%$, the $\mathrm{YI}$ is 0.71 , and the Kappa value is 0.718 . When the two are combined in series, the sensitivity, specificity, positive predictive value, negative predictive value, accuracy, and YI and Kappa values are $63.65 \%, 95.54 \%, 93.18 \%, 70.14 \%, 78.56 \%, 0.61$, and 0.58 , respectively, as shown in Figure 4 and Table 6.

4.5. Comparison of Four Groups of Serum Concentrations. The levels of serum WIBG, ZNF706, and GABARAPL2 in the four groups are shown in Table 7. In this experiment, they were compared. The serum ZNF706 levels of the four groups were compared. The serum ZNF706 levels of the ERA group, the NERA group, the CTD group, and the healthy group were $[(86.35 \pm 2.96), \quad(60.37 \pm 2.19)$, $(52.64 \pm 3.91)$, and $(47.35 \pm 2.64) \mathrm{ng} / \mathrm{L}]$, the ZNF706 level in the serum of the ERA group was the highest, which was significantly higher than that of the NERA group, CTD group, and healthy group, and the difference was statistically significant $(p<0.05)$. The levels of WIBG in the four groups were $[(1539.12 \pm 116.42),(1233.67 \pm 99.34),(993.14 \pm 85.91$ ), and $(424.52 \pm 30.96) \mathrm{ng} / \mathrm{L}]$. The serum WIBG level in the ERA group was the highest, which was significantly higher than that in the NERA group, CTD group, and healthy group, and the difference was statistically significant $(p<0.05)$. The levels of GABARAPL2 in the four groups were $[(39.47 \pm 2.86), \quad(28.63 \pm 1.76), \quad(20.68 \pm 0.94)$, and $(15.03 \pm 0.55) \mathrm{ng} / \mathrm{ml}$. The level of GABARAPL2 in the serum of the ERA group was the highest, which was significantly higher than that of the NERA group, CTD group, and healthy group, and the difference was statistically significant $(p<0.05)$, as shown in Figure 5 and Table 7.

4.6. Rheumatoid Arthritis-Related Drugs. With the rapid development of economy and society, people's lives and eating habits have changed. According to the monitoring data of Zhongkang $\mathrm{CMH}$, among the five major rheumatoid arthritis drugs, Chinese herbal preparations have the largest market share. The market size reached 22.3 billion yuan in 2019, accounting for $37.4 \%$, and it is expected to reach 25.6 billion yuan by 2020 . The estimated compound annual growth rate (2016-2019) is 6.1\%. As rheumatoid arthritis is a chronic disease, antirheumatic drugs (DMARD) to improve this condition are also getting more and more attention, and the market performance of DMARD also shows this. The market size of DMARD has expanded from RMB 9 billion in 2016 to 2019. From 11.7 billion yuan (18.1\%), it will reach 12.9 
TABLE 8: Proportion of drugs in recent years.

\begin{tabular}{|c|c|c|c|c|c|}
\hline Drugs & 2016 & 2017 & 2018 & 2019 & $2020(E)$ \\
\hline Plant preparation & $41.60 \%$ & $40.20 \%$ & $38.80 \%$ & $37.40 \%$ & $35.30 \%$ \\
\hline Biologics & $20.80 \%$ & $20.80 \%$ & $20.80 \%$ & $20.80 \%$ & $20.80 \%$ \\
\hline Anti-inflammatory drugs & $17.60 \%$ & $17.90 \%$ & $18.30 \%$ & $18.10 \%$ & $17.50 \%$ \\
\hline Antirheumatic drugs & $10.70 \%$ & $11.20 \%$ & $13.70 \%$ & $14.10 \%$ & $15.80 \%$ \\
\hline Glucocorticoid & $6.10 \%$ & $6.22 \%$ & $6.39 \%$ & $6.63 \%$ & $6.86 \%$ \\
\hline
\end{tabular}

billion yuan by 2020. Among the five main categories of therapeutic drugs, the compound annual growth rate (20162020 ) is the highest, reaching $11.8 \%$, as shown in Figure 6 and Table 8.

\section{Conclusion}

New disease research methods are helping the experiment find new evidence for the diagnosis of diseases. Protein chip technology and the combination of computer technology and biomedicine will have more applications and developments. Autoantibodies ZNF706, GABARAPL2, and WIBG can identify early RA and provide a new direction for understanding the mechanism of early disease and the mechanism of disease development. At the same time, the experiment showed that the expression of certain markers in the early and early stages of rheumatoid arthritis is quite different. The discovery of these markers enables the experiment to show that the pathological process of rheumatoid arthritis is different in the early and late stages. At the same time, their application will inevitably improve the accuracy of rheumatoid arthritis and will also play an important role and improve the early diagnosis of rheumatoid arthritis. Some of these indicators will also provide more clues and guidance for the development of new drugs.

At present, there is consensus on the treatment of rheumatoid arthritis at home and abroad. Once a patient is diagnosed with rheumatoid arthritis, effective disease-improving drugs should be used as soon as possible to control disease progression and prevent or reduce bone damage. This is conducive to improving the prognosis of the disease, and early diagnosis is the basis for early treatment. The diagnostic criteria currently revised by the American Academy of Rheumatology in 1987 are mainly based on clinical manifestations, X-ray examinations, and serum rheumatoid factor (RF) tests. When the diagnostic criteria for imaging are met, the patient will experience bone destruction. RF has low sensitivity and specificity in other autoimmune diseases (such as systemic lupus erythematosus and healthy elderly) and also has a certain positive rate. There are some shortcomings in clinical application, which delays the optimal treatment time and increase the disability rate. The above criteria are not helpful for the early diagnosis of RA. Therefore, in recent years, research has become a research hotspot in finding simple and easy-to-detect laboratory detection methods with high sensitivity or specificity to improve the early diagnosis rate.

Early diagnosis of rheumatoid arthritis is a hot spot in recent years, because early diagnosis and treatment of rheu- matoid arthritis can effectively prevent the progression of the disease and delay or even prevent the process of bone destruction. The current ACR/EULAU 2010 RA diagnostic criteria for early diagnosis of rheumatoid arthritis have been significantly improved. Among them, RF and CCP antibodies are used for diagnosis. However, in clinical practice, there are some difficulties in diagnosing patients with negative RF and CCP antibodies. For some patients who are highly suspected but have not yet reached the diagnostic criteria, more information is needed to support the diagnosis. At the same time, the current ACR/EULAU2010 RA diagnostic criteria still has a certain false positive rate for the early diagnosis of rheumatoid arthritis, which also requires more data to help diagnosis.

\section{Data Availability}

The data that support the findings of this study are available from the corresponding author upon reasonable request.

\section{Conflicts of Interest}

The author(s) declare(s) that they have no conflicts of interest.

\section{References}

[1] L. Buisman, J. Luime, M. Oppe, J. M. W. Hazes, and M. P. M. H. Rutten-van Mölken, "A five-year model to assess the early cost-effectiveness of new diagnostic tests in the early diagnosis of rheumatoid arthritis," Arthritis Research \& Therapy, vol. 18, no. 1, article 1020, pp. 135-A359, 2016.

[2] H. Y. Wu, A. Filer, I. Styles, and H. Dehghani, "Development of a multi-wavelength diffuse optical tomography system for early diagnosis of rheumatoid arthritis: simulation, phantoms and healthy human studies," Biomedical Optics Express, vol. 7, no. 11, pp. 4769-4786, 2016.

[3] Y. H. Cheon and S. I. Lee, "Impact of early diagnosis on functional preservation in patients with rheumatoid arthritis: the early bird catches the worm," Korean Journal of Internal Medicine, vol. 32, no. 4, pp. 634-635, 2017.

[4] M. Vojdanian, H. Ahmadi, A. R. Jamshidi et al., "The antimigraine effects of M2000 ( $\beta$-D-mannuronic acid) on a patient with rheumatoid arthritis: case report," Current Clinical Pharmacology, vol. 12, no. 2, pp. 127-130, 2017.

[5] Q. Feng, Y. Li, N. Wang et al., "A biomimetic nanogenerator of reactive nitrogen species based on battlefield transfer strategy for enhanced immunotherapy," Small (Weinheim an der Bergstrasse, Germany), vol. 16, no. 25, article e2002138, 2020. 
[6] G. Orsolini, C. Caimmi, O. Viapiana et al., "Titer-dependent effect of anti-citrullinated protein antibodies on systemic bone mass in rheumatoid arthritis patients," Calcified Tissue International, vol. 101, no. 1, pp. 17-23, 2017.

[7] V. F. A. M. Derksen, S. Ajeganova, L. A. Trouw et al., "Rheumatoid arthritis phenotype at presentation differs depending on the number of autoantibodies present," Annals of the Rheumatic Diseases, vol. 76, no. 4, pp. 716-720, 2017.

[8] L. Mayordomo, M. L. Velloso, C. Almeida, L. M. JimenezLiñan, C. Gomez-Cano, and J. L. Marenco, "AB0221 Predictive value of power Doppler ultrasonography (PDUS) in the diagnosis of very early rheumatoid arthritis," Annals of the Rheumatic Diseases, vol. 75, Supplement 2, pp. 973.3-97973, 2016.

[9] S. M. Al-Shaer, "Role of CT scan in early diagnosis of temporomandibular joint injury in patients with rheumatoid arthritis," The Journal of Contemporary Dental Practice, vol. 21, no. 7, pp. 769-775, 2020.

[10] M. Elhoseny, K. Shankar, and J. Uthayakumar, "Intelligent diagnostic prediction and classification system for chronic kidney disease," Scientific Reports, vol. 9, no. 1, article 46074, p. 9583, 2019.

[11] M. Peña and F. Rondón, “AB0224 Role of anti-RA33 as a biomarker for diagnosis in Colombian patients with rheumatoid arthritis," Annals of the Rheumatic Diseases, vol. 75, Supplement 2, pp. 974.3-97974, 2016.

[12] H. Song and M. Brandt-Pearce, "Range of influence and impact of physical impairments in long-haul DWDM systems," Journal of Lightwave Technology, vol. 31, no. 6, pp. 846-854, 2013.

[13] P. R. Lage-Hansen, H. Lindegaard, S. Chrysidis, and L. Terslev, "The role of ultrasound in diagnosing rheumatoid arthritis, what do we know? An updated review," Rheumatology International, vol. 37, no. 2, article 3587, pp. 179-187, 2017.

[14] L. B. Solnes, K. M. Jones, S. P. Rowe et al., "Diagnostic value of18F-FDG PET/CT versus MRI in the setting of antibodyspecific autoimmune encephalitis," Journal of Nuclear Medicine, vol. 58, no. 8, pp. 1307-1313, 2017.

[15] L. Ji, X. Deng, Y. Geng, Z. Song, and Z. Zhang, "The additional benefit of ultrasonography to 2010 ACR/EULAR classification criteria when diagnosing rheumatoid arthritis in the absence of anti-cyclic citrullinated peptide antibodies," Clinical Rheumatology, vol. 36, no. 2, article 3465, pp. 261-267, 2017.

[16] L. Ji, G. Li, Y. Xu, W. Zhou, and Z. Zhang, "Early prediction of rheumatoid arthritis by magnetic resonance imaging in the absence of anti-cyclic citrullinated peptide antibodies and radiographic erosions in undifferentiated inflammatory arthritis patients: a prospective study," International Journal of Rheumatic Diseases, vol. 18, no. 8, pp. 859-865, 2015.

[17] S. Chaudhary, D. Dutta, M. Kumar et al., "Vitamin D supplementation reduces thyroid peroxidase antibody levels in patients with autoimmune thyroid disease: an open-labeled randomized controlled trial," Indian Journal of Endocrinology \& Metabolism, vol. 20, no. 3, article 179997, pp. 391-398, 2016.

[18] S. Arora, A. Rafiq, and M. Jolly, "Management of rheumatoid arthritis: review of current guidelines," Journal of Arthroscopy \& Joint Surgery, vol. 3, no. 2, pp. 45-50, 2016.

[19] Z. C. Ozdemir, O. Bor, E. C. Dinleyici, and E. Kiral, "Plasmapheresis in a child with cold antibody autoimmune hemolytic anemia: case report," Turkish Archives of Pediatrics/Türk Pediatri Arşivi, vol. 52, no. 3, pp. 169-172, 2017.
[20] J. Y. Mao, X. H. Zhao, H. Dai, X. J. Gao, and L. L. Lu, "Rheumatoid arthritis involving tibialis posterior tendon: study on the early diagnostic value of power Doppler ultrasonography and comparison with surgery," Zhonghua Yi Xue Za Zhi, vol. 96, no. 41, pp. 3311-3314, 2016.

[21] E. L. Nasonov, Y. A. Olyunin, and A. M. Lila, "Rheumatoid arthritis: the problems of remission and therapy resistance," Scientific and practical rheumatology, vol. 56, no. 3, pp. 263271,2018

[22] C. Strehl, L. Maurizi, S. Hermann et al., “AB0014nanoparticles as MRI contrast agent for early diagnosis of RA: effects of amino-PVA-coated SPIONS on CD4+ T cell activity," Annals of the Rheumatic Diseases, vol. 75, Supplement 2, pp. 901-901, 2016.

[23] J. A. Schwartz, I. Prado, J. Misamore et al., "An HIV gp120CD4 immunogen does not elicit autoimmune antibody responses in cynomolgus macaques," Clinical \& Vaccine Immunology, vol. 23, no. 7, pp. 618-627, 2016.

[24] V. Hamuryudan, H. Direskeneli, I. Ertenli et al., "Direct and indirect healthcare costs of rheumatoid arthritis patients in Turkey," Clinical \& Experimental Rheumatology, vol. 34, no. 6, pp. 1033-1037, 2016.

[25] M. P. Brown, P. Hissaria, A. H. Hsieh, C. Kneebone, and W. Vallat, "Autoimmune limbic encephalitis with anticontactin-associated protein-like 2 antibody secondary to pembrolizumab therapy," Journal of Neuroimmunology, vol. 305, pp. 16-18, 2017.

[26] N. M. Mcgrath and C. P. Turner, "Isolated gluteal and paravertebral muscle weakness due to anti-3-hydroxy-3-methylglutaryl-coenzyme a reductase antibody-associated necrotizing autoimmune myopathy," Muscle \& Nerve, vol. 54, no. 1, pp. 150-152, 2016.

[27] B. I. Gavrilă, C. Ciofu, and V. Stoica, "Biomarkers in rheumatoid arthritis, what is new?," Journal of Medicine \& Life, vol. 9, no. 2, pp. 144-148, 2016.

[28] J. A. Lansita, K. M. Mease, H. Qiu, T. Yednock, S. Sankaranarayanan, and S. Kramer, "Nonclinical development of ANX005: a humanized anti-C1q antibody for treatment of autoimmune and neurodegenerative diseases," International Journal of Toxicology, vol. 36, no. 6, pp. 449462, 2017.

[29] S.-B. Tsai, Y.-Z. Xue, P.-Y. Huang et al., "Establishing a criteria system for green production," Proceedings of the Institution of Mechanical Engineers, Part B: Journal of Engineering Manufacture, vol. 229, no. 8, pp. 1395-1406, 2015.

[30] X. Xu, D. Cao, Y. Zhou, and J. Gao, "Application of neural network algorithm in fault diagnosis of mechanical intelligence," Mechanical Systems and Signal Processing, vol. 141, article $106625,2020$. 